\title{
Diferencias en la producción investigadora en tesis y artículos de los profesores funcionarios de Psicología en España en función del sexo
}

\author{
José Alonso Olivas-Ávila ${ }^{1 *}$, Bertha Musi-Lechuga ${ }^{2}$, Alejandro Guillén-Riquelme ${ }^{2}$ y Ángel Castro ${ }^{3}$ \\ ${ }^{1}$ Universidad Autónoma de Ciudad Juárez, (México) ${ }^{2}$ Universidad de Granada (España), ${ }^{3}$ Universidad de Zaragoza (España)
}

\begin{abstract}
Resumen: El objetivo de esta investigación es analizar si existen diferencias en la producción científica de los profesores funcionarios más productivos de la Psicología española en función del sexo. Para ello, se seleccionaron los 610 profesores funcionarios más productivos de las distintas áreas de la Psicología y se analizó su producción en artículos incluidos en revistas del Journal Citation Reports, en citas recibidas en esos artículos y en tesis doctorales dirigidas. En los resultados obtenidos se observa que la producción de los hombres es mayor que la de las mujeres en los tres indicadores analizados, con diferencias variables en función del área de la Psicología. En la discusión se analizan las causas de estas diferencias, prestando especial atención a la tardía incorporación de la mujer a la universidad española. Palabras clave: producción científica; investigación; sexo; profesores funcionarios; psicología; universidad española.
\end{abstract}

\section{Introducción}

Uno de los desafíos fundamentales a los que se enfrenta la universidad española tras la implantación del Espacio Europeo de Educación Superior (EEES) es conseguir un aumento tanto en la calidad de las investigaciones que se realizan, como en la visibilidad y transferencia de los resultados obtenidos (Bermúdez, Castro, Sierra y Buela-Casal, 2009; Carbonell y Calvó, 2009; Conferencia de Rectores de Universidades Españolas [CRUE], 2011). Fruto de esta necesidad, en los últimos años se ha publicado un gran número de estudios e informes en los que se analiza la productividad de la universidad española, desde distintas perspectivas y comprendiendo a todos los agentes implicados en la enseñanza e investigación universitaria. Se analiza la productividad de profesores, en criterios como los artículos publicados en revistas incluidas en el Journal Citation Reports (JCR) (MusiLechuga, Olivas-Ávila y Castro, 2011a; Musi-Lechuga, Olivas-Ávila, Portillo-Reyes y Villalobos-Galvis, 2005; OlivasÁvila y Musi-Lechuga, 2010a) o en tesis doctorales dirigidas (Musi-Lechuga, Olivas-Ávila y Castro, 2011b; Olivas-Ávila y Musi-Lechuga, 2010b), la relación que existe entre la dirección de tesis doctorales y la publicación de artículos y se analiza el número y el tipo de citaciones que reciben los profesores e investigadores (Buela-Casal, 2010; Buela-Casal y Zych, 2010; Robinson-García, Delgado-López-Cozar, y Torres-Salinas, 2011; Villar, 2011), para mejorar los criterios e indicadores de selección del profesorado (Betz, 2010; BuelaCasal y Sierra, 2007; Zych, 2011) e incluso, según criterios asociados a la calidad (Romero, 2009). También se analiza la productividad de los profesores dentro de los programas de

* Dirección para correspondencia [Correspondence address]: José Alonso Olivas-Ávila. Programa de Psicología. Universidad Autónoma de Ciudad Juárez. Av. Universidad y H. Colegio Militar s/n. Zona Chamizal, 32300, Ciudad Juárez, Chihuahua (México).

E-mail: jolivas@uacj.mx
Title: Psychology professors research production differences in theses and articles in Spain by gender.

Abstract: The aim of this work is to analyze whether differences exist in the scientific research of the most productive professors in Spanish psychology based on their gender. For this purpose, we analyzed the production of a sample of the 610 most productive professors in the different areas of psychology in articles published in journals of the Journal Citation Reports, by citations of these articles and in doctoral dissertations directed. The results show that the production of males higher than is for women in the indicators analyzed, with variable differences based on psychology areas. The discussion examines the causes of these differences, focusing in the later entry of women to the Spanish university.

Key words: scientific production; research; sex; professors; psychology; spanish university.

postgrado en los que participan (Musi-Lechuga, Olivas-Ávila y Buela-Casal, 2009) y dentro de las universidades en las que desempeñan su trabajo, creando así los rankings de universidades españolas que han florecido en los últimos años (Buela-Casal, Bermúdez, Sierra, Quevedo-Blasco y Castro, 2009, 2010) y que han dado pie a relacionar la productividad científica con la financiación que reciben las universidades (Buela-Casal, Bermúdez, Sierra, Quevedo-Blasco, GuillénRiquelme et al., 2010; Musi-Lechuga, Olivas-Ávila, GuillenRiquelme y Castro, 2011).

$\mathrm{El}$ análisis de las diferencias en la productividad en investigación en función del sexo es una corriente muy relevante dentro de este nuevo campo de estudio, no solo por el aspecto bibliométrico y académico, sino también por su contenido social. En las dos últimas décadas son muchos los estudios, tanto a nivel nacional como internacional, en los que se ha comparado la producción de investigadores hombres y mujeres. La mayoría ha llegado a la conclusión de que la producción de las mujeres es inferior a la de los hombres (Abramo, D'Angelo y Caprasecca, 2009; Aksens, Rorstad, Piro y Siversten, 2011; Lariviere, Vignola-Gagne, Villeneuve, Gelinas y Gingras, 2011; Maz-Machado, et al, 2011; TorresSalinas, Muñoz-Muñoz y Jiménez-Contreras, 2011). Las causas que se dan para explicar estas diferencias son muy variadas. Algunas tienen que ver con los acontecimientos vitales, como puede ser la maternidad. Hunter y Leahey (2010) afirman que después de tener un hijo desciende la productividad de los investigadores, sea cual sea su sexo, pero que el descenso es mucho más pronunciado entre las mujeres. La edad también es una variable relevante, pues hay autores que defienden que durante ciertos periodos de la vida de las mujeres se observa un descenso en la producción, sobre todo a partir de los 40 años (Lariviere et al., 2011; Mauleón y Bordons, 2006).

Una visión llamativa, de alto contenido social, es la que aportan Kaplan et al. (1996), quienes concluyen que las mu- 
jeres investigadoras presentan tasas más bajas de productividad científica porque dedican más tiempo y preocupación a la docencia y al cuidado de los pacientes, en las disciplinas médicas, con lo que poseen menos tiempo para dedicar a la investigación. De una manera u otra, como afirma Leahey (2006), las diferencias en la productividad científica en función del sexo constituyen un complejo puzle de difícil solución.

En España, como afirman Sierra, Buela-Casal, Bermúdez y Santos-Iglesias (2009), diversos organismos e instituciones han analizado la distribución de hombres y mujeres dentro del profesorado universitario. Las distintas normativas de selección del profesorado universitario abogan por la igualdad de oportunidades a todos los candidatos, pero esto no garantiza que haya una proporción igualitaria de hombres y mujeres. Curiosamente, en 2010 se cumplió cien años de la Real Orden que permitió el acceso de las mujeres a las aulas universitarias en España en condiciones de igualdad. A fecha de 2011, según el Ministerio de Educación (2011), el 54.2\% del total de estudiantes universitarios en España son mujeres y su presencia es mayoritaria en todos los niveles de formación universitaria, pues suponen el $54.1 \%$ de los estudiantes de máster y alrededor del 51\% entre los de doctorado. La presencia de la mujer es predominante en todas las áreas de conocimiento, con excepción de las técnicas, y su presencia es masiva en titulaciones de Ciencias de la Salud $(72.1 \%$ de mujeres) y en Ciencias Sociales y Jurídicas (64.1\%), entre las que se encuentra la Psicología. Pero conforme se avanza en el escalafón de los colectivos implicados en la educación universitaria, la presencia de las mujeres decrece. Según la CRUE (2011), las mujeres representan solo el 36.2\% del Personal Docente e Investigador de las universidades españolas, siendo mujeres el $33.7 \%$ de los profesores funcionarios. El dato se agrava cuando se hace referencia a los Catedráticos de Universidad, entre los que solo el 14.3\% son mujeres. Además, otras instituciones destacan la menor presencia de mujeres en puestos directivos, tales como rectorados, decanatos o vicedecanatos (Ministerio de Educación y Ciencia, 2007).

La explicación más frecuente a este descenso de la proporción de mujeres conforme aumenta la posición académica, se fundamenta en la tardía incorporación de éstas a la universidad. Este hecho conlleva la necesidad de tiempo para que esas personas, que desde hace más de diez años son mayoría en las aulas, puedan desarrollar sus carreras científicas e investigadoras, llegando así a puestos de profesores funcionarios y de gestión de las universidades (Aksens et al., 2011; Torres-Salinas et al., 2011), además de lograr una mejora en su producción científica, asemejándose e incluso superando a la de los hombres (Bordons, Morillo, Fernández y Gómez, 2003).

Por lo visto anteriormente, se plantea el objetivo de la presente investigación que es analizar si existen diferencias en la producción científica de los profesores funcionarios de la Psicología española en función del sexo. En primera instancia se deseaba analizar si existían diferencias a lo largo de toda la trayectoria vital de esos profesores, pero debido a que de esa manera se podía estar perjudicando a las mujeres, que comenzaron a entrar en la universidad española de forma más tardía, se ha incluido un análisis del periodo 2000 2010, para analizar también la producción en un periodo más reciente y constatar si existen diferencias con la producción de toda la carrera académica.

\section{Método}

\section{Participantes}

Para la selección de la muestra se tomó como referencia el artículo de Musi-Lechuga et al. (2005). A partir de él, se seleccionó la muestra de los profesores funcionarios con mayor producción en artículos de cada área de la Psicología española en documentos extraídos de la Web of Science (WoS). Finalmente, se contó con 610 profesores (aproximadamente cien por cada una de las seis áreas de conocimiento de la Psicología en España).

\section{Unidades de análisis}

- Tesis doctorales dirigidas: obtenidas de la base de Tesis Españolas Ordenadas (TESEO). Se buscó en primer lugar sin acotar temporalmente $\mathrm{y}$, posteriormente, las tesis dirigidas entre el año 2000 y el 2010. La búsqueda se realizó entre el 18 y el 28 de junio de 2010.

- Número de artículos de revistas indexadas en el JCR: se utilizó la WoS. Se buscó, en primer lugar, sin acotar temporalmente por fecha de publicación de los artículos y, posteriormente, aquellos que se publicaron entre el año 2000 y el 2010. Las búsquedas se llevaron a cabo entre los meses de noviembre de 2009 y marzo de 2010.

- Número de citas: se utilizó la WoS para localizar el número de citas recibidas por cada profesor de la muestra, tanto entre los años 2000 y 2010, como sin acotar temporalmente. Las búsquedas se llevaron a cabo entre los meses de noviembre de 2009 y marzo de 2010.

- Áreas de conocimiento de la Psicología en España: Psicología Básica, Psicología de la Educación, Metodología de las Ciencias del Comportamiento, Personalidad Evaluación y Tratamiento Psicológicos, Psicobiología y Psicología Social.

\section{Materiales}

- Base de datos TESEO.

- Base de datos Web of knowledge (WoK).

\section{Procedimiento}

Tras la selección de la muestra, en función de lo establecido por Musi-Lechuga et al. (2005), se pasó a determinar la producción en artículos indexados en el JCR, las citas recibidas por estos artículos en revistas incluidas en el JCR y el número de tesis doctorales dirigidas. Para la búsqueda de las 
tesis doctorales dirigidas, se utilizó el buscador TESEO, incluyendo los apellidos de los profesores, en las distintas combinaciones posibles, en el campo de director. En el caso de los artículos, se utilizó la WoS incluyendo, de nuevo, varias combinaciones del nombre en el campo autor, así como la inicial o iniciales del nombre. Las combinaciones de los apellidos se emplearon para evitar posibles pérdidas de registros porque los apellidos estuviesen escritos en un formato diferente al uso normal, por ejemplo, unidos mediante guiones. Desde esta misma plataforma se puede acceder a las citas que otros autores hacen en artículos en revistas indexadas en el JCR. Los resultados se restringieron a artículos procedentes de España y se incluyeron artículos, revisiones y reimpresiones. En primera instancia no se acotó temporalmente la búsqueda, con lo que se incluyeron todos los resultados obtenidos para cada profesor en los tres indicadores. En una segunda etapa, se acotó temporalmente la búsqueda, analizando los registros de artículos, citas recibidas y tesis doctorales dirigidas entre los años 2000 y 2010.

Las búsquedas fueron realizadas por dos investigadores de forma independiente. Este mecanismo permitía observar el grado de consenso entre ambas búsquedas para detectar posibles fallos en el proceso. No se encontraron grandes discrepancias entre los investigadores. Posteriormente, se realizaron los análisis estadísticos de forma independiente para los datos que incluyen toda la trayectoria y para los datos de registros entre los años 2000 y 2010. Para la redacción de este manuscrito se siguieron las recomendaciones de Ramos-Álvarez, Moreno-Fernández, Valdés-Conroy y Catena (2008).

\section{Resultados}

En primer lugar, se analizó la distribución por sexos. De los 610 participantes, el $37.9 \%$ fueron mujeres $(n=231)$ y el $62.1 \%$ restante, hombres $(n=379)$. Seguidamente, se pasó a analizar cómo era la distribución en función del sexo en cada una de las áreas de conocimiento. Los resultados se muestran en la Figura 1.

En la figura se observa que la distribución por sexos varía notablemente. En Psicobiología las diferencias son prácticamente inexistentes, mientras que en otras áreas, como en Psicología Básica, las diferencias son mayores. Estas diferencias en la distribución por sexo de las distintas áreas son estadísticamente significativas con un $\chi_{(5 ; n=610)}^{2}=14.77(p=$ $.01)$.

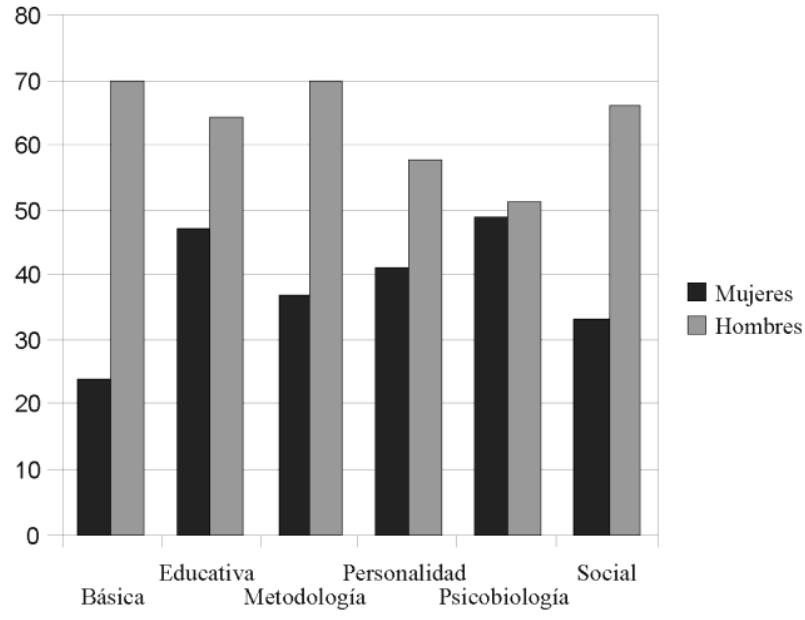

Figura 1. Frecuencia de hombres y mujeres entre los cien profesores con mayor producción de cada área de conocimiento de la psicología.

Una vez establecida la distribución por sexo en cada una de las áreas cabe analizar la producción en los diversos indicadores presentados. Así pues, en primer lugar se analizó el número de artículos publicados en revistas indexadas en el JCR en toda la trayectoria y en los últimos diez años. Para ello, se realizó una comparación de medias por sexo para cada una de las áreas y otra para el total. Los resultados, tanto de los artículos publicados en toda la trayectoria, como los de los últimos diez años, se recogen en la Tabla 1.

Se puede observar que en la producción de la trayectoria completa hay diferencias estadísticamente significativas $(p<$ .05) para la muestra global y en las áreas de Metodología de las Ciencias del Comportamiento, en Personalidad, Evaluación y Tratamiento Psicologicos y en Psicobiología. Al analizar las diferencias en la producción de los últimos diez años, las diferencias se mantienen únicamente en el área de Metodología de las Ciencias del Comportamiento y en el total de la muestra, aunque en este caso el tamaño del efecto es insignificante.

Los mismos análisis se realizaron para el caso de las tesis doctorales dirigidas, tanto en los últimos diez años como en toda la trayectoria. Los resultados se recogen en la Tabla 2 y constatan las diferencias existentes en las áreas de Metodología de las Ciencias del Comportamiento, Psicología Educativa, Psicología Social y Psicobiología, a lo largo de toda la trayectoria. Al realizar los análisis para las tesis dirigidas en los últimos diez años, las diferencias únicamente se mantienen en el total de la muestra, con un tamaño del efecto insignificante, y en el área de Piscología Social. 
Tabla 1. Comparación por sexo del número de artículos publicados en revistas indexadas en el Journal Citation Reports (JCR) de los 100 profesores funcionarios con mayor producción de cada área de conocimiento de la psicología, en toda la trayectoria profesional y entre los años 2000 a 2010.

\begin{tabular}{|c|c|c|c|c|c|c|c|c|c|}
\hline \multirow[b]{2}{*}{ Área } & \multirow[b]{2}{*}{ Sexo } & \multicolumn{3}{|c|}{ Artículos JCR totales } & \multicolumn{5}{|c|}{ Artículos JCR años 2000-2010 } \\
\hline & & Media (DT) & t-student (g.l.) & $p$ & $\begin{array}{l}\text { Tamaño del } \\
\text { efecto }\end{array}$ & Media (DT) & t-student (g.l.) & $p$ & $\begin{array}{l}\text { Tamaño del } \\
\text { efecto }\end{array}$ \\
\hline \multirow{2}{*}{ Básica } & Hombres & $15.41(14.77)$ & \multirow{2}{*}{$0.79(92)^{*}$} & \multirow{2}{*}{.434} & \multirow{2}{*}{0.18} & $10.49(11.11)$ & \multirow{2}{*}{$0.94(92)^{*}$} & \multirow{2}{*}{.351} & \multirow{2}{*}{0.22} \\
\hline & Mujeres & $12.84(11.43)$ & & & & $8.20(8.38)$ & & & \\
\hline \multirow{2}{*}{ Educativa } & Hombres & $6.09(7.37)$ & \multirow{2}{*}{$0.86(109)^{*}$} & \multirow{2}{*}{.395} & \multirow{2}{*}{0.17} & $4.47(6.11)$ & \multirow{2}{*}{$0.76(109)^{*}$} & \multirow{2}{*}{.448} & \multirow{2}{*}{0.15} \\
\hline & Mujeres & $5.00(5.27)$ & & & & $3.64(4.78)$ & & & \\
\hline \multirow{2}{*}{ Metodología } & Hombres & $15.38(12.34)$ & \multirow{2}{*}{$2.54(105)^{*}$} & \multirow{2}{*}{.013} & \multirow{2}{*}{0.51} & $10.71(9.98)$ & \multirow{2}{*}{$1.98(105)^{*}$} & \multirow{2}{*}{.050} & \multirow{2}{*}{0.40} \\
\hline & Mujeres & $9.58(9.08)$ & & & & $6.97(8.05)$ & & & \\
\hline \multirow{2}{*}{ Personalidad } & Hombres & $22.74(17.19)$ & \multirow{2}{*}{$2.05(97)^{*}$} & \multirow{2}{*}{.043} & \multirow{2}{*}{0.42} & $15.91(14.93)$ & \multirow{2}{*}{$1.68(97)^{*}$} & \multirow{2}{*}{.096} & \multirow{2}{*}{0.34} \\
\hline & Mujeres & $16.71(9.45)$ & & & & $11.62(8.26)$ & & & \\
\hline \multirow{2}{*}{ Psicobiología } & Hombres & $30.10(23.92)$ & \multirow{2}{*}{$2.02(98)^{*}$} & \multirow{2}{*}{.046} & \multirow{2}{*}{0.40} & $16.92(14.26)$ & 175 (98)* & 083 & 035 \\
\hline & Mujeres & $20.37(24.30)$ & & & & $11.20(18.18)$ & & & \\
\hline Social & Hombres & $8.68(9.03)$ & 101 (97)* & 314 & 023 & $6.56(8.06)$ & 0.75 (97)* & 457 & 016 \\
\hline & Mujeres & $6.87(5.08)$ & & & & $5.39(4.92)$ & & & \\
\hline Total & Hombres & $15.65(16.45)$ & $2.65(531.62) * *$ & 008 & 021 & $10.46(11.70)$ & $265(51371) * *$ & 008 & 022 \\
\hline & Mujeres & $12.27(14.45)$ & & & & $7.99(10.79)$ & & & \\
\hline
\end{tabular}

Nota Básica = Psicología Básica; Educativa = Psicología Evolutiva y de la Educación; Metodología = Metodología de las Ciencias del Comportamiento; Personalidad $=$ Personalidad, Evaluación y Tratamiento Psicológicos; Social= Psicología Social.

* Se asumen varianzas iguales, en función de la prueba de Levene.

** No se asumen varianzas iguales, en función de la prueba de Levene.

Tabla 2. Comparación por sexo del número de citas recibidas en artículos publicados en revistas indexadas en el Journal Citation Reports (JCR) de los 100 profesores funcionarios con mayor producción de cada área de conocimiento de la psicología, en toda la trayectoria profesional y entre los años 2000 a 2010.

\begin{tabular}{|c|c|c|c|c|c|c|c|c|c|}
\hline \multirow[b]{2}{*}{ Área } & \multirow[b]{2}{*}{ Sexo } & \multicolumn{4}{|c|}{ Citas totales } & \multicolumn{4}{|c|}{ Citas de artículos 2000-2010 } \\
\hline & & Media (DT) & t-Student (g.l.) & $p$ & $\begin{array}{c}\text { Tamaño } \\
\text { del efecto }\end{array}$ & Media (DT) & t-Student (g.l.) & $p$ & $\begin{array}{l}\text { Tamaño } \\
\text { del efecto }\end{array}$ \\
\hline \multirow{2}{*}{ Básica } & Hombres & $147.20(207.93)$ & \multirow{2}{*}{$0.45(92)^{*}$} & \multirow{2}{*}{.656} & \multirow{2}{*}{0.10} & 72.07 (116.40) & \multirow{2}{*}{$.837(92)^{*}$} & \multirow{2}{*}{.405} & \multirow{2}{*}{0.22} \\
\hline & Mujeres & $124.60(240.60)$ & & & & $49.56(11.83)$ & & & \\
\hline \multirow{2}{*}{ Educativa } & Hombres & $21.41(35.35)$ & \multirow{2}{*}{$0.11(109)^{*}$} & \multirow{2}{*}{.909} & \multirow{2}{*}{0.02} & $14.70(30.97)$ & \multirow{2}{*}{$-0.022(109)^{*}$} & \multirow{2}{*}{.983} & \multirow{2}{*}{0.00} \\
\hline & Mujeres & $20.56(42.89)$ & & & & $14.84(41.10)$ & & & \\
\hline \multirow{2}{*}{ Metodología } & Hombres & $81.96(132.04)$ & \multirow{2}{*}{$3.02(86.84)^{* *}$} & \multirow{2}{*}{.003} & \multirow{2}{*}{0.47} & $48.90(85.07)$ & \multirow{2}{*}{$2.63(87.60)^{* *}$} & \multirow{2}{*}{.010} & \multirow{2}{*}{0.41} \\
\hline & Mujeres & $30.37(38.53)$ & & & & $19.92(25.46)$ & & & \\
\hline \multirow{2}{*}{ Personalidad } & Hombres & $125.23(133.71)$ & \multirow{2}{*}{$0.87(97)^{*}$} & \multirow{2}{*}{.386} & \multirow{2}{*}{0.18} & $73.19(110.08)$ & \multirow{2}{*}{$0.82(97)^{*}$} & \multirow{2}{*}{.411} & \multirow{2}{*}{0.17} \\
\hline & Mujeres & $105.07(78.81)$ & & & & $57.90(55.39)$ & & & \\
\hline \multirow{2}{*}{ Psicobiología } & Hombres & $408.94(533.47)$ & \multirow{2}{*}{$1.62(98)^{*}$} & \multirow{2}{*}{.110} & \multirow{2}{*}{0.32} & $168.35(223.44)$ & $1.73(98) *$ & 088 & 035 \\
\hline & Mujeres & $250.24(443.77)$ & & & & $94.61(203.02)$ & & & \\
\hline Social & Hombres & $56.96(105.83)$ & $0.79(97) *$ & .430 & 0.17 & $41.50(75.24)$ & $0.94(97) *$ & .350 & 0.21 \\
\hline & Mujeres & $41.23(45.79)$ & & & & & & & \\
\hline & Hombres & $129.19(259.28)$ & & & & $65.52(123.75)$ & & & \\
\hline Total & Mujeres & $100.64(238.20)$ & $1.39(515$ & .166 & 0.11 & $46.06(109.92)$ & $2.02(527.55)^{* *}$ & .044 & 0.16 \\
\hline
\end{tabular}

Nota: Básica = Psicología Básica; Educativa = Psicología Evolutiva y de la Educación; Metodología = Metodología de las Ciencias del Comportamiento; Personalidad $=$ Personalidad, Evaluación y Tratamientos Psicológicos; Social= Psicología Social.

* Se asumen varianzas iguales, en función de la prueba de Levene.

** No se asumen varianzas iguales, en función de la prueba de Levene.

Por último, se analizó el número de citas recibidas por los artículos publicados en revistas indexadas en el JCR (las citas fueron realizadas en artículos del JCR). Los resultados obtenidos se pueden observar en la Tabla 3. Solo se encontraron diferencias estadísticamente significativas en el área de Metodología de las Ciencias del Comportamiento, tanto a lo largo de toda la trayectoria como en los últimos diez años, aunque en ambos casos el tamaño del efecto es moderado. 


\section{Discusión}

A partir de los resultados obtenidos en esta investigación, se puede concluir que existen diferencias importantes en la producción científica de los profesores funcionarios de la Psicología española en función del sexo. Las diferencias se pueden observar desde el primero de los datos contrastados, pues se obtuvo que el $62.1 \%$ de los profesores funcionarios con mayor producción de la Psicología en España son hombres, mientras que las mujeres suponen el $37.9 \%$. Este dato se corresponde con la distribución general de mujeres entre el profesorado funcionario de la universidad española, pues- to que, como ya se expuso en la introducción de este artículo, según el estudio de la CRUE (2011), las mujeres representan el 36.2\% del total de profesores funcionarios en España. Si se observa la distribución de los profesores funcionarios en Psicología en función del sexo, ofrecida por MusiLechuga et al. (2005), a partir de los datos de la Dirección General de Universidades y del Consejo de Coordinación Universitaria, se puede constatar que las mujeres suponen el $41.4 \%$ del total de profesores, un porcentaje ligeramente mayor a la media nacional de todas las áreas de conocimiento.

Tabla 3. Comparación por sexo del número de tesis dirigidas de los 100 profesores funcionarios con mayor producción de cada área de conocimiento de la psicología, en toda la trayectoria profesional y entre los años 2000 a 2010.

\begin{tabular}{|c|c|c|c|c|c|c|c|c|c|}
\hline \multirow[b]{2}{*}{ Área } & \multirow[b]{2}{*}{ Sexo } & \multicolumn{3}{|c|}{ Tesis totales } & \multicolumn{5}{|c|}{ Tesis $2000-2010$} \\
\hline & & Media (DT) & $t$-Student (g.l.) & $p$ & $\begin{array}{l}\text { Tamaño } \\
\text { del efecto }\end{array}$ & Media (DT) & t-Student (g.l.) & $p$ & $\begin{array}{c}\text { Tamaño } \\
\text { del efecto }\end{array}$ \\
\hline \multirow{2}{*}{ Básica } & Hombres & $3.57(4.85)$ & \multirow{2}{*}{$1.483(92)^{*}$} & \multirow{2}{*}{.142} & \multirow{2}{*}{0.35} & $2.16(2.41)$ & \multirow{2}{*}{$1.49(92)^{*}$} & \multirow{2}{*}{.141} & \multirow{2}{*}{0.35} \\
\hline & Mujeres & $2.04(2.79)$ & & & & $1.36(2.00)$ & & & \\
\hline \multirow{2}{*}{ Educativa } & Hombres & $5.74(5.06)$ & \multirow{2}{*}{$2.79(109)^{*}$} & \multirow{2}{*}{.006} & \multirow{2}{*}{0.54} & $3.23(3.24)$ & \multirow{2}{*}{$1.96(109)^{*}$} & \multirow{2}{*}{.053} & \multirow{2}{*}{0.38} \\
\hline & Mujeres & $3.13(4.52)$ & & & & $2.02(3.10)$ & & & \\
\hline \multirow{2}{*}{ Metodología } & Hombres & $2.57(2.73)$ & \multirow{2}{*}{$0.27(105)^{*}$} & \multirow{2}{*}{.020} & \multirow{2}{*}{0.06} & $1.36(1.49)$ & \multirow{2}{*}{$-0.76(48.42)^{* *}$} & \multirow{2}{*}{.452} & \multirow{2}{*}{-0.18} \\
\hline & Mujeres & $2.39(3.89)$ & & & & $1.74(2.84)$ & & & \\
\hline \multirow{2}{*}{ Personalidad } & Hombres & $5.77(4.71)$ & \multirow{2}{*}{$1.34(97)^{*}$} & \multirow{2}{*}{.184} & \multirow{2}{*}{0.27} & $3.40(3.10)$ & \multirow{2}{*}{$0.81(97)^{*}$} & \multirow{2}{*}{.423} & \multirow{2}{*}{0.16} \\
\hline & Mujeres & $4.55(4.19)$ & & & & $2.93(2.61)$ & & & \\
\hline \multirow{2}{*}{ Psicobiología } & Hombres & $3.67(3.19)$ & \multirow{2}{*}{$2.81(91.67)^{* *}$} & \multirow{2}{*}{.006} & \multirow{2}{*}{0.56} & $2.63(2.58)$ & $1.95(98) *$ & .054 & 0.39 \\
\hline & Mujeres & $2.10(2.34)$ & & & & $1.73(1.95)$ & & & \\
\hline Social & Hombres & $5.51(6.65)$ & $3.35(96.89)^{* *}$ & .00 & 0.57 & $3.09(3.63)$ & $2.92(90.93)^{* *}$ & .004 & 0.53 \\
\hline & Mujeres & $2.23(3.12)$ & & & & $1.39(2.12)$ & & & \\
\hline & Hombres & $4.46(4.90)$ & & & & $2.62(2.90)$ & & & \\
\hline Total & Muieres & $281(368)$ & $4.71(580.15)^{* *}$ & 0 & 0.37 & $1.92(2.54)$ & $3.10(532.40)^{* *}$ & .002 & 0.25 \\
\hline
\end{tabular}

Nota : Básica = Psicología Básica; Educativa = Psicología Evolutiva y de la Educación; Metodología = Metodología de las Ciencias del Comportamiento; Personalidad = Personalidad, Evaluación y Tratamiento Psicológicos; Social= Psicología Social.

* Se asumen varianzas iguales, en función de la prueba de Levene

** No se asumen varianzas iguales, en función de la prueba de Levene

En la distribución por áreas, se observó que entre los profesores con mayor producción en Psicobiología eran muy similares por sexo, pero que en otras áreas como Psicología Básica, Metodología de las Ciencias del Comportamiento y Psicología Social, las diferencias son mayores, pues la distribución por sexo de los profesores con mayor producción tiene un $70 \%$ de hombres y el $30 \%$ de mujeres. Según los datos ofrecidos por Musi-Lechuga et al. (2005), estas tres áreas son las que presentan una mayor presencia masculina en el profesorado funcionario, rondando el 65\% de hombres y el 35\% de mujeres, así que es comprensible que la distribución sea más dispar aún cuando se hace referencia a los que tienen mayor produción.

Las diferencias observadas se constatan en cada uno de los criterios analizados. En los artículos publicados en revistas del JCR, se obtuvo que los hombres han publicado una media de 3.5 artículos más que las mujeres, tanto a lo largo de toda su carrera investigadora como, y eso es sorprendente, en los últimos diez años, en los que se ha producido un gran avance de la mujer en la universidad española. Las diferencias son mayores en unas áreas que en otras, destacando Psicobiología, en la que los hombres publican una media de diez artículos más que las mujeres en toda su trayectoria, o en Metodología de las Ciencias del Comportamiento, en la que se observa una diferencia de seis artículos en toda la trayectoria y de cuatro artículos más publicados por los hombres en los últimos diez años. Las áreas de Metodología y de Psicología Básica tienen una presencia mucho mayor de hombres que de mujeres entre los profesores funcionarios.

En cuanto a las tesis doctorales dirigidas por los profesores funcionarios con mayor producción de la Psicología en España, se obtuvo que los hombres habían dirigido una media mayor de tesis doctorales, tanto en toda su trayectoria, como en los últimos diez años. Las diferencias eran mayores 
en áreas como la Psicología Evolutiva y de la Educación (2.5 tesis dirigidas más de media los hombres en toda su trayectoria), Psicología Social (3.5 tesis más dirigidas por los hombres en toda su trayectoria y 1.5 más en los últimos diez años). En las citas obtenidas por los artículos publicados por estos profesores en revistas del JCR se encontraron diferencias menos relevantes que en los dos apartados anteriores.

Las grandes diferencias obtenidas en la producción científica de los profesores en función del sexo permiten extraer varias lecturas. En primer lugar y como ya se ha puesto de manifiesto en la introducción de este trabajo, la literatura existente sobre el tema resalta que la productividad en investigación de las mujeres suele ser inferior a la de los hombres (Abramo et al., 2009; Kaplan et al., 1996; Leahey, 2006; Mauleón y Bordons, 2006; Maz-Machado et al., 2011) y que éstas, debido a su reciente entrada en la universidad, necesitan algo más de tiempo para situarse entre la élite investigadora (Torres-Salinas et al., 2011). Esto es comprensible y parece estar asumido en los autores de trabajos similares, tanto en España como en otros países, pero, según los resultados obtenidos en esta investigación, si se tienen en cuenta solo los últimos diez años, la producción investigadora de las mujeres también es menor, sobre todo en artículos publicados en revistas del JCR y en tesis doctorales dirigidas; dos de los indicadores más relevantes para medir la productividad científica de profesores e investigadores a nivel nacional e internacional. Prpić (2002) puso de manifiesto que las diferencias en la productividad científica de los investigadores en función del sexo se habían incrementado en los cinco años anteriores a realizar su estudio, en los que las mujeres habían publicado una media de dos artículos menos que los hombres en revistas incluidas en el JCR. Sin embargo, Bermúdez

\section{Referencias}

Abramo, G., D'Angelo, C.A. y Caprasecca, A. (2009). Gender differences in research productivity: A bibliometric analysis of the Italian academic systems. Scientometrics, 79, 517-539.

Aksens, D.W., Rorstad, K., Piro, F. y Siversten, G. (2011). Are female researchers less cited? A large-scale study of Norwegian scientists. Journal of the American Society for Information Science and Technology, 62, 628-636.

Bermúdez, M.P., Castro, A., Sierra, J.C. y Buela-Casal, G. (2009). Análisis descriptivo transnacional de los estudios de doctorado en el EEES. Revista de Psicodidáctica, 14, 193-221.

Bermúdez, M.P., Guillén-Riquelme, A., Gómez-García, A., Quevedo-Blasco, R., Sierra, J.C. y Buela-Casal, G. (2011). Análisis del rendimiento en el doctorado en función del sexo. Educación XX1, 14, 17-33.

Betz, B. (2010). Análisis de las fortalezas institucionales para la contratación de investigadores. Aula Abierta, 38, 65-74.

Bordons, M., Morillo, F., Fernández, M.T. y Gómez, I. (2003). One step further in the production of bibliometric indicators at the micro level: Differences by gender and professional category of scientists. Scientometrics, 57, 159-173.

Buela-Casal, G. (2010). Índices de impacto de las revistas científicas e indicadores para medir el rendimiento de los investigadores. Revista de Psicodidáctica, 15, 3-19.

Buela-Casal, G., Bermúdez, M.P., Sierra, J.C., Quevedo-Blasco, R. y Castro, A. (2009). Ranking de 2008 en productividad en investigación de las universidades públicas españolas. Psicothema, 21, 304-312.

Buela-Casal, G., Bermúdez, M.P., Sierra, J.C., Quevedo-Blasco, R. y Castro, A. (2010). Ranking de 2009 en investigación de las universidades públi- et al. (2011) concluyeron que no existían diferencias entre hombres y mujeres en las tasas de éxito del doctorado, es decir, en terminar la formación doctoral con la conclusión de la tesis. Esto puede considerarse como un avance hacia una producción más igualitaria y hacia una mayor presencia y visibilidad de las mujeres investigadoras.

En otros estudios se concluye que la producción de las mujeres aumenta conforme aumenta su posición en el sistema universitario. Bordons et al. (2003) afirman que, en áreas como Farmacia o Recursos Naturales, se observa una creciente feminización, que hace que se espere una mayor presencia y visibilidad de las mujeres y su trabajo en un futuro cercano. Desde otra óptica, Peñaranda Ortega, Quiñones Vidal y López García, (2005) han encontrado una diferencia casi nula con datos recientes, en los que por medio de un análisis bibliométrico de la revista Anales de Psicología, “...el porcentaje de autores hombres y mujeres es similar pese al número de posibles autores, en mayoría hombres, en la Psicología española". Lo cierto es que, como afirman Sierra et al. (2009), al evaluar la productividad en función del número de sexenios de investigación obtenidos, se está evaluando también la edad y el sexo de los profesores; en edades más altas hay un mayor porcentaje de varones en el profesorado universitario, por la tardía incorporación de la mujer. Habrá que esperar algo más de tiempo, a que aumente, sobre todo, ese $14.3 \%$ que suponen las mujeres entre el total de Catedráticos de Universidad (CRUE, 2011), para evaluar si la menor producción de las mujeres es un hecho estructural en la universidad española, o si bien es su todavía baja presencia en las esferas académicas más altas la que explica esa menor producción.

cas españolas. Psicothema, 22, 171-179.

Buela-Casal, G., Bermúdez, M.P., Sierra, J.C., Quevedo-Blasco, R., GuillénRiquelme, A. y Castro, A. (2010). Relación de la productividad y eficiencia en investigación con la financiación de las comunidades autónomas españolas. Psicothema, 22, 924-931.

Buela-Casal, G. y Sierra, J.C. (2007). Criterios, indicadores y estándares para la acreditación de profesores titulares y catedráticos de universidad. Psicothema, 19, 537-551.

Buela-Casal, G. y Zych, I. (2010). Analysis of the relationship between the number of citations and the quality evaluated by experts in psychology journals. Psicothema, 22, 270-276.

Carbonell, X. y Calvó, N. (2009). Las revistas españolas de Psicología: Cómo elegir la revista donde publicar. Anales de Psicología, 25, 209-216.

Conferencia de Rectores de Universidades Españolas (2011). La universidad española en cifras 2010. Recuperado el 20 de mayo de 2011 de http://www.crue.org/Publicaciones/UEC.html.

Hunter, L.A. y Leahey, E. (2010). Parenting and research productivity: New evidence and methods. Social Studies of Science, 40, 433-451.

Kaplan, S.H., Sullivan, L.M., Dukes, K.A., Phillips, C.F., Kelch, R.P. y Schaller, J.G. (1996). Sex differences in academic advancement.- Results for a national study of pediatricians. New England Journal of Medicine, 335, 1282-1289.

Lariviere, V., Vignola-Gagne, E., Villeneuve, C., Gelinas, P. y Gingras, Y. (2011). Sex differences in research funding, productivity and impact: an analysis of Quebec university professors. Scientometrics, 87, 483-498.

Leahey, E. (2006). Gender differences in productivity.- Research specializa- 
tion as a missing link. Gender \& Society, 20, 754-780.

Mauleón, E. y Bordons, M. (2006). Productivity, impact and publication habits by gender in the area of Materials Science. Scientometrics, 66, 199218.

Maz-Machado, A., Gutiérrez-Arenas, M.P., Bracho-López, R., Jiménez Franjul, N., Adamuz-Povedano, N. y Torralbo, R. (2011). Producción científica en Ciencias Sociales de las mujeres en Andalucía (2003-2007). Aula Abierta, 39, 63-72.

Ministerio de Educación (2011). Datos y cifras del sistema universitario español. Curso 2010-2011. Recuperado el 25 de mayo de 2011 de http://www.educacion.gob.es/educacion/universidades/estadisticasinformes/novedades.html.

Ministerio de Educación y Ciencia (2007). Académicas en cifras 2007. Recuperado el 20 de abril de 2011 de http://www.micinn.es/ciencia/umyc/files/2007-academicas-cifras.pdf.

Musi-Lechuga, B., Olivas-Ávila, J.A. y Buela-Casal, G. (2009). Producción científica de los programas de doctorado en Psicología Clínica y de la Salud en España. International Journal of Clinical and Health Psychology, 9, 161-173.

Musi-Lechuga, B., Olivas-Ávila, J.A. y Castro, A. (2011a). Productividad de los programas de doctorado en psicología con Mención de Calidad en artículos de revistas incluidas en el Journal Citation Reports. Psicothema, 23, 343-348.

Musi-Lechuga, B., Olivas-Ávila, J.A. y Castro, A. (2011b). Productividad en tesis de los programas de doctorado en Psicología con Mención de Calidad. Revista Mexicana de Psicología, 28, 93-100.

Musi-Lechuga, B., Olivas-Ávila, J.A., Guillen-Riquelme, A. y Castro, A. (2011). Relación entre productividad y eficiencia de los programas de doctorado en Psicología. Revista Latinoamericana de Psicología, 43, 297-305.

Musi-Lechuga, B., Olivas-Ávila, J.A., Portillo-Reyes, V. y Villalobos-Galvis, F. (2005). Producción de los profesores funcionarios de Psicología en España en artículos de revistas con factor de impacto de la Web of Science. Psicothema, 17, 539-548.

Olivas-Ávila, J.A. y Musi-Lechuga, B. (2010a). Análisis de la producción de los profesores funcionarios de Psicología en España en artículos de re- vistas en la Web of Science. Psicothema, 22, 909-916.

Olivas-Ávila, J.A. y Musi-Lechuga, B. (2010b). Producción en tesis doctorales de los profesores funcionarios de Psicología en España más productivos en la Web of Science. Psicothema, 22, 917-923.

Peñaranda Ortega, M., Quiñones Vidal, E. y López García, J.J. (2005). Veinte años de Anales de Psicología: Una revista con raíces académicas (1984-2004). Anales de Psicología, 21, 181-198.

Prpić, K. (2002). Gender and productivity differentials in science. Scientometrics, 55, 27-58.

Ramos-Álvarez, M.M., Moreno-Fernández, M.M., Valdés-Conroy, B. y Catena, A. (2008). Criteria of the peer review process for publication of experimental and quasi-experimental research in Psychology: A guide for creating research papers. International Journal of Clinical and Health Psychology, 8, 751-764.

Robinson-García, N., Delgado-López-Cozar, E. y Torres-Salinas, D. (2011). Cómo comunicar y diseminar información científica en Internet para obtener mayor visibilidad e impacto. Aula Abierta, 39, 41-50.

Romero Medina, A. (2009). Evolución de la calidad y difusión de una revista científica española de Psicología: Anales de Psicología, 25 volúmenes anuales publicados (1984-2009). Anales de Psicología, 25, 181-198.

Sierra, J.C., Buela-Casal, G., Bermúdez, M.P. y Santos-Iglesias, P. (2009). Diferencias por sexo en los criterios y estándares de productividad científica y docente en profesores funcionarios en España. Psicothema, 21, 124-132.

Torres-Salinas, D., Muñoz-Muñoz, A.M. y Jiménez-Contreras, E. (2011). Bibliometric analysis of the situation of female researchers in the fields of social sciences and law in Spain. Revista Española de Documentación Cientifica, 34, 11-28.

Villar, A. (2011). El "eigenfactor": un nuevo y potente instrumento bibliométrico para evaluar la investigación. Aula Abierta, 39, 85-96.

Zych, I. (2011). Comparación de los criterios para la acreditación de profesores contratados y funcionarios. Aula Abierta, 39, 51-62.

(Artículo recibido: 10-07-2011, revisión: 12-10-2011, aceptado: 22-10-2011) 